\title{
GESTIÓN SOSTENIBLE DE LOS HERITAGE SITES CULTURALES: PROPUESTA DE UN MODELO
}

\author{
Silvia Fresneda Fuentes* \\ Universidad de Sevilla \\ https://orcid.org/0000-0001-9828-2832 \\ Pilar de Fuentes Ruíz \\ Universidad de Sevilla \\ https://orcid.org/0000-0001-9338-0628 \\ Antonio Lobo Gallardo \\ Universidad de Sevilla \\ https://orcid.org/0000-0003-4021-6044
}

\section{RESUMEN}

La gestión sostenible de los bienes de patrimonio histórico demanda sistemas de control de gestión que contemplen sus particularidades. El objetivo de este trabajo es el diseño de un modelo de control holístico, contingente e integrador a emplear por los responsables públicos y/o privados de su gestión. Se ha realizado una profunda revisión bibliográfica de la literatura. El modelo propuesto no pretende ser universal, sino un punto de referencia para su adaptación a las particularidades propias de cada bien. Incluye indicadores que permitirán medir y valorar el logro de los objetivos.

Palabras clave: Sistema de control, gestión sostenible; turismo sostenible; heritage sites.

\section{Sustainable management of cultural heritage sites: a model suggestion}

\section{ABSTRACT}

The requirement of a sustainable management of the historical heritage sites demands the design of management control models that contemplate the particularities of those sites, beyond the architectural ones. The goal of this paper is the design of a holistic, contingent

Fecha de recepción: 11 de marzo de 2019

Fecha de aceptación: 31 de octubre de 2020

* Departamento de Contabilidad y Economía Financiera. Facultad de Ciencias Económicas y Empresariales. Universidad de Sevilla. Avenida Ramón y Cajal, 1.41018 SEVILLA. E-mail: fresneda@us.es, pfuentes@ us.es, alobo@us.es 
and integrator model to be used by the public and/or private managers for the sustainable management. To achieve this, a thorough bibliographic review of the specialized literature has been carried out. This model does not pretend to be universal but rather a point of reference for its adaptation to the particularities of each site.

Keywords: Control system; sustainable management; sustainable tourism; heritage sites.

\section{INTRODUCCIÓN}

El turismo es una de las principales fuentes de ingresos en la economía española. En 2016 este sector aportó un 11,2\% al Producto Interior Bruto nacional y generó un 13\% del empleo (INE, 2017)).

La Organización Mundial del Turismo (OMT) estima que en torno al 37\% de las cifras anuales del sector turístico se derivan del turismo cultural y considera que está en continuo crecimiento (WTO, 2019). No obstante, la Comisión Europea del Turismo en conjunción con la OMT ya en 2005 alertan del peligro que supone para el patrimonio histórico-cultural su crecimiento descontrolado y anima a diversificar la oferta (ETC/WTO, 2005). En este sentido, Hernández (2002) señala que las buenas perspectivas económicas han convertido a este turismo en un producto que, si no se sabe gestionar bien, puede incluso poner en peligro su propia supervivencia e identidad, causar problemas, tales como deslocalización, pérdida de "cultura", dependencia económica y degradación del ecosistema. En consecuencia, todas las actividades turísticas de cualquier motivación necesitan ser sostenibles (UNESCO, 2014).

El desarrollo sostenible se basa en (Brundtland, 1987; UNESCO, 2014):

- Planificación holística y toma de decisiones estratégicas.

- Preservación de los procesos ecológicos.

- Protección del patrimonio humano y biodiversidad.

- Crecimiento sostenible a largo plazo.

El turismo sostenible es aquel que respeta tanto a la gente local como al turista cultural, al bien cultural y al medio ambiente. Busca proporcionar a la gente unas vacaciones excitantes y que a la vez beneficie a los habitantes del lugar (UNWTO; UNESCO, 2014). Puede ser definido como "El turismo que tiene plenamente en cuenta las repercusiones actuales y futuras, económicas, sociales y medioambientales para satisfacer las necesidades de los visitantes, de la industria, del entorno y de las comunidades anfitrionas” (UNWTO).

Los principios de sostenibilidad se refieren a los aspectos medioambiental, económico y sociocultural del desarrollo turístico, habiéndose de establecer un equilibrio adecuado entre esas tres dimensiones para garantizar su sostenibilidad a largo plazo (Bramwell y Lane, 1993; UNESCO, 2014; Brito Rodríguez y Cànoves Valiente, 2019) 
todo ello encuadrable dentro de un sistema más global que incluya indicadores más cuantificables que permitan la comparabilidad.

El desarrollo sostenible del turismo exige la participación informada de todos los agentes relevantes o grupos de interés, así como un liderazgo político firme para lograr una colaboración amplia y establecer un consenso (Landorf, 2009; UNESCO, 2014). El logro de un turismo sostenible es un proceso continuo de gestión y requiere un seguimiento constante de sus incidencias, para introducir las medidas preventivas o correctivas que resulten necesarias. Además, debe reportar también un alto grado de satisfacción a los turistas y representar para ellos una experiencia significativa, que los haga más conscientes de los problemas de la sostenibilidad y fomente en ellos unas prácticas turísticas sostenibles.

No obstante lo anterior, no son muchos los estudios que analizan cuestiones relacionadas con la gobernanza de los destinos turísticos y el turismo sostenible y la necesidad de adaptar estas nuevas formas de gobierno a contextos y situaciones específicas (Bramwell y Lane, 2011).

La necesidad de una gestión sostenible también se ha puesto de manifiesto en el turismo cultural. La consideración del patrimonio cultural desde un punto de vista económico pone de manifiesto la necesidad de conjugar actuaciones encaminadas a su protección y conservación con otras que desarrollen y potencien su papel económico (UNESCO, 2014), por ello, entendemos que su gestión se debe afrontar desde una visión empresarial sostenible (Lara de Vicente y López-Guzmán Guzmán, 2004). Coincidimos con Ho y McKercher (2004) en que los bienes de patrimonio histórico han de considerarse como productos turísticos con cuyo lanzamiento al mercado se pretende suministrar una experiencia enriquecedora que satisfaga las necesidades de los turistas. Por ello, cada vez más, las investigaciones desarrolladas se han centrado en la comprensión del comportamiento del turista y en su gestión estratégica (Garrod y Fyall, 2000). Así, los gestores se encuentran con la tarea de adaptar la demanda a través de un acercamiento entre patrimonio y turista, explicándoles las características particulares del patrimonio tangible e intangible desde una perspectiva amena, sencilla, atractiva y cómoda.

En relación al turismo cultural, España posee una posición privilegiada pues tiene un extenso patrimonio histórico, artístico y lingüístico. En concreto, cuenta con 47 bienes culturales de la UNESCO, lo que la sitúa a nivel mundial en segundo lugar después de Italia que tiene 53 bienes culturales.

En países como Italia, Francia, Reino Unido y España la mayor parte de los Heritage Sites (HS) son propiedad de Administraciones Públicas (AAPP), por ello, los mecanismos de gestión y los sistemas de información implantados en los HS han estado determinados por las decisiones tomadas desde la esfera política y han sido similares a los empleados por la AAPP. Los cambios acontecidos en la filosofía de gestión de las AAPP derivados de movimientos conocidos como Nueva gestión pública (NGP) y Nueva gobernanza pública (NGobP) se han venido trasladando a los HS.

En este contexto, el objetivo de este trabajo es diseñar un modelo holístico, contingente e integrador a emplear por los responsables públicos y /o privados para la gestión sostenible de los HS. 
Para alcanzar dicho objetivo se ha realizado una profunda revisión bibliográfica de la literatura especializada en modelos de gestión de HS con la que se han identificado las variables que condicionan la gestión sostenible de los mismos.

Además de esta introducción, el trabajo se estructura en 6 epígrafes. Del segundo al quinto se incluyen los resultados de la revisión bibliográfica realizada sobre nueva gestión pública, nueva gobernanza pública y gestión sostenible de los HS. En el sexto presentamos el modelo propuesto. En el último se exponen las conclusiones y las líneas futuras de investigación.

\section{NUEVA GESTIÓN PÚBLICA Y MEDICIÓN DEL RENDIMIENTO}

En países como Italia y España, la gestión de gran parte del patrimonio cultural es llevada a cabo por las AAPP. Por ello, durante mucho tiempo, se ha realizado según la lógica burocrática imperante en dichas administraciones, propia de un entorno estable, lento y con escasos cambios.

En los años noventa del pasado siglo en la administración pública de algunos países (Italia, España, Francia, Reino Unido, Australia, etc.) se comienza a implementar un cambio en el modelo de gestión burocrática tradicional, coherente con un entorno de rápido cambio, en el que los ciudadanos demandan mejores servicios y menos impuestos. Las prácticas de gestión de las empresas privadas se trasladan al sector público por isomorfismo mimético (DiMaggio y Powell, 1983) dando lugar al movimiento denominado NGP (Hood, 1995). Se produce la denominada "empresarialización" del sector público, bajo la creencia que la aplicación de la filosofía de gestión importada del sector privado mejoraría los resultados.

Como resultado de todo ello las AAPP abandonan su papel tradicional de simples prestadores de servicios públicos. A tal efecto se crean organizaciones de diversa naturaleza -organizaciones no gubernamentales, fundaciones, entidades públicas, empresas públicas, etc.- que compiten por la obtención de financiación y por ser los elegidos para prestar los servicios públicos. En este escenario el rol asumido por las AAPP es el de estimular, facilitar, organizar y controlar cómo estas organizaciones están prestando los servicios.

En este cuasi-mercado se introducen los criterios de eficacia, eficiencia y economicidad, la competencia, y el ciudadano pasa a ser considerado como un stakeholder y no como un mero pagador de impuestos (Paddison y Walmsley, 2018).

La medición del rendimiento se convierte en una tarea a resolver en las AAPP. No obstante, la introducción de la lógica de gestión empresarial se ha centrado básicamente en la utilización de modelos enfocados al control de costes (Donato, 2011). Donato (2011) considera que después de 20 años de la introducción de la filosofía de la NGP en Italia el modelo burocrático no ha sido eliminado totalmente de las AAPP, ambos conviven, lo cual produce incoherencias y contradicciones que han representado un obstáculo importante en la mejora de gestión de las AAPP. Esta misma circunstancia se reproduce en el caso español.

En relación al sistema de medida de rendimiento en el sector cultural, hemos de señalar que son muchas las publicaciones relacionadas con la gestión y los sistemas de información de los museos (Asuaga y Rausell, 2006) pero pocas las que han tomado 
como sujeto de estudio los bienes histórico-patrimoniales (Millar, 1989; Laws, 1998; Lafortune et al., 1999).

Schuster (1996) señala que el establecimiento de un conjunto de medidas de rendimiento en el sector tiene una doble complejidad, la naturaleza del sector -cultura- y la naturaleza de las entidades que generalmente lo gestionan -pública y no lucrativa-, caracterizadas por ser burocráticas e inflexibles (Zan, 2006).

Ashworth y Page (2011) ponen de manifiesto la escasez de estudios que evalúen cómo el discurso neoliberal que ha dado lugar a la NGP ha afectado a la toma de decisiones, sistemas de control y su implementación en el turismo urbano (Baraldi, 2014).

Baraldi (2014) además señala que esta ausencia de estudios es aún más notable en aquellos países que no son de corte anglosajón. En este sentido, Donato (2011) indica que el modelo de gestión de la NGP de las instituciones culturales que se ha concebido, proyectado y aplicado en los países anglosajones y, por tanto, no se debe importar directamente a países como Italia, España o Francia, puesto que las características del patrimonio cultural y las propias políticas culturales son diferentes.

En los países anglosajones, salvo en UK, el resto -USA, Australia y Nueva Zelandason países de una relativa reciente creación, que no tienen la tradición cultural de los europeos cuyo modelo es de tipo "micro" orientado o enfocado en la gestión de instituciones individuales que las considera como un único sujeto que no tiene vínculos con otros sujetos o instituciones culturales.

Por otro lado, en la Unión Europea, los países del norte se han enfocado en los procesos de producción cultural y los del sur en poner en valor el extenso patrimonio cultural existente. Esto ha hecho que las políticas comunitarias hayan favorecido la producción cultural y el desarrollo de la creatividad y hayan dejado a un lado la financiación de los HS. Sin embargo, en Italia, Francia y España, las instituciones culturales tienen siglos de tradición, están en simbiosis con el territorio y mantienen vínculos con otras instituciones. Hay una interacción lugar e identidad que se manifiesta en las características sociales, culturales, ambientales y paisajísticas, arquitectónicas, de lenguaje y hábitos que caracterizan el lugar. Por esta razón, en Italia, el modelo de NGP "micro" debe ser un poco más amplio y abordarse desde un enfoque multi-scala (Donato, 2011).

Son diversos los estudios que se han realizado en Italia a este respecto (Badia et al., 2012; Badia y Donato, 2013; Baraldi, 2014) pero escasos los realizados en España (Pons et al., 2011; Bonet y Donato, 2011; Ulldemolins y Arostegui, 2013).

\section{NUEVA GOBERNANZA PÚBLICA Y PARTICIPACIÓN ACTIVA DE LOS GRUPOS DE INTERÉS}

La NGobP también surge en los años 90 como resultado de las deficiencias y carencias detectadas en la NGP con carácter general y, más concretamente, en los países europeocontinentales.

La lógica de la NGobP se fundamenta en los conceptos de participación -derivada de la orientación hacia el exterior- y capacitación. Sus elementos fundamentales se basan en la capacidad de construir una red de relaciones entre las AAPP y los interlocutores sociales (Badia Et Al., 2012; Runya et al., 2015). Concretamente: 
- Gestión basada en redes, en el caso en que no se pueda identificar un único sujeto responsable de dicha gestión. Las redes se pueden configurar de diversa forma (Longo, 2005): (1) todos los sujetos de la red se sitúan en el mismo plano, no detentan particulares poderes o control los unos sobre los otros (red paritaria); (2) una institución goza de poderes para ejercer importantes funciones de dirección y control sobre el resto de integrantes de la red, pero sin obedecer a un claro principio jerárquico top-down (red pivotante) y (3) una administración pública recoge en su "unitariedad" un sistema de otras organizaciones que dependen de ella.

- Búsqueda de vías alternativas a los mecanismos de competencia introducidos por la NGP, en los casos en que éstos no sean la solución.

- Introducción de instrumentos que permitan la consecución de un consenso externo, que vayan más allá de la búsqueda de la eficiencia, eficacia y economicidad y que se fundamenten en la tutela y el respeto de los valores básicos de la comunidad, dando, cuando sea posible, voz y voto a los ciudadanos y a todos los grupos de interés.

Entre los principales agentes relacionados con los HS destacamos los siguientes (Du Cros et al., 2005; Fresneda y Lobo, 2014; UNESCO, 2014):

a.- La Administración Pública. Además de ser propietaria de los HS, es responsable de la aprobación de leyes de patrimonio y protección, de la fijación de políticas culturales, establecimiento de los principios y guías para la conservación y uso del patrimonio histórico-cultural, desarrollo e implementación de estrategias de desarrollo del turismo, inversiones en transportes e infraestructuras públicas, etc.

b.- El sector privado, compuesto por el tejido industrial, comercial y hotelero de la zona, el cual además de financiar determinados proyectos, es el que concentra la mayor parte de la inversión en hoteles y canaliza la casi totalidad de los flujos turísticos a través de las agencias de viajes.

c.- Organizaciones no gubernamentales, locales, nacionales e internacionales (tales como la UNESCO), que pueden facilitar fondos, servir de apoyo, investigar en diversos temas, otorgar menciones especiales tales como Bienes Patrimonio de la Humanidad (WHS -world heritage sites-), etc.

d.- El turista cultural, como "cliente" de este producto. La gestión responsable y sostenible de las entidades públicas ha puesto de manifiesto la relevancia de los usuarios y la necesidad de medir su satisfacción, tarea harto difícil (Colbert, 2014).

e.- La comunidad en general, como beneficiaria y además contribuyente.

En este sentido, como señala Landorf (2009) la gestión de los HS requiere de la participación activa de todos estos grupos de interés, en particular la de la comunidad local, no obstante, con carácter general su participación suele limitarse al establecimiento de "planes formales de naturaleza política" más que a una participación activa en el desarrollo de las estrategias. Por otro lado, como señalan (Aas et al., 2005) la participación equitativa de todos estos grupos de interés en la gestión de los HS es teórica (Tan et al., 2018). 


\section{LA GESTIÓN SOSTENIBLE DE LOS HERITAGE SITES}

Dada su relevancia y la necesidad de obtener resultados positivos, se ha observado una preocupación creciente tanto de los responsables de la gestión de los HS como de la comunidad investigadora por analizar los factores a considerar en la "explotación sostenible del patrimonio cultural" (Fresneda y Lobo, 2014; UNESCO, 2014).

No obstante, la propia naturaleza tan fragmentada de la industria, su globalidad, el elevado grado de competencia, la débil relación entre oferta y demanda, entre otros, han motivado una ausencia de una aproximación holística e integradora en la gestión sostenible de los HS (Welford et al., 1999).

Hasta 1993, su gestión se había enfocado, básicamente, en la toma de decisiones sobre la conservación del aspecto tangible dejando a un lado el intangible y la actividad del visitante (Landorf, 2009; Badia et al., 2012; Rodwell, 2012; Wilson y Boyle, 2006) . A ello se une el hecho de que el aspecto cultural debe ir de forma conjunta con el aspecto de gestión económica (Badia et al., 2012; Su et al., 2018).

\section{Figura 1}

\section{GESTIÓN INTEGRAL DE LA CALIDAD EN UN HERITAGE SITE}

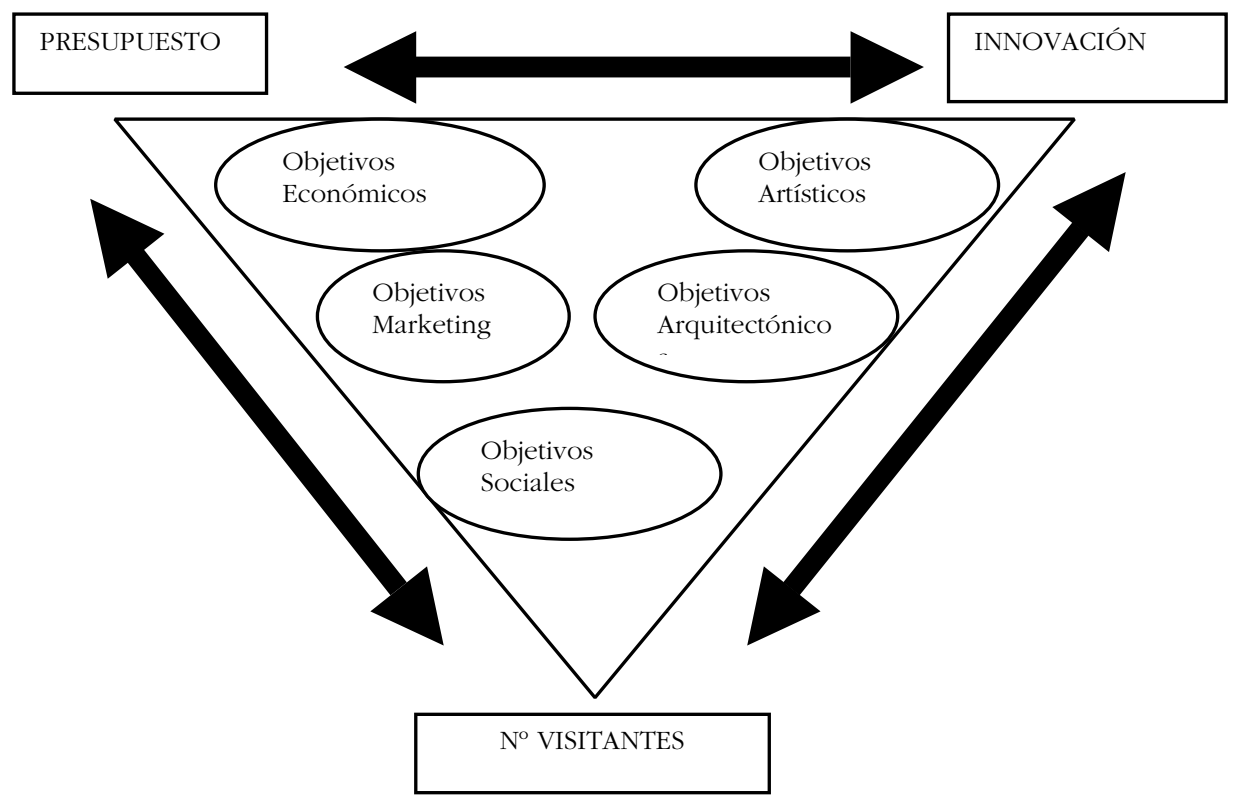

Fuente: Adaptado de Tajtáková (2006).

Según (Alazaizeh et al., 2016; Alazaizeh et al., 2018) en el enfoque convencional de la gestión se ignoraban los turistas, pues el foco de atención se centraba en el bien en sí. Actualmente, la tendencia global ha evolucionado desde el desarrollo de un producto 
basado en las exposiciones y en la educación a un enfoque más orientado al visitante, a sus preferencias y la calidad de experiencia personal (Alexandros y Jaffry, 2005).

En este contexto, la calidad y su administración se convierten en variables estratégicas a tener en cuenta en el turismo cultural ya que va a suponer una adecuada gestión de los recursos culturales para así transformarlos en productos (Castro León, 2005). La gestión integral de la calidad de un WHS requiere, por un lado, de una cooperación entre los diferentes grupos de interés implicados en el proceso, tanto públicos como privados, y por otro, que el propio órgano gestor sepa tener en cuenta las particularidades y los conflictos de intereses de cada colectivo (Fresneda y Lobo, 2014). Además de ello, debe tener en cuenta tres pilares fundamentales que están en permanente conflicto: las restricciones presupuestarias, la innovación y la afluencia de público. Por otro lado, al no ser el lucro el objetivo a perseguir, el éxito de su gestión está ligado, tanto cuantitativa como cualitativamente, a los servicios brindados a la comunidad con los recursos disponibles, siendo la satisfacción de las necesidades de dicha comunidad uno de los objetivos primordiales (Kaplan y Norton, 2001; Kaplan y Norton, 2001a; UNESCO, 2014). Por otro lado, está el patrimonio cultural tangible de incalculable valor económico que hay que conservar y preservar del deterioro que supone no sólo el paso del tiempo, sino la afluencia de visitantes.

Landorf (2009) indica que la inclusión de un bien histórico en la lista de los WHS de la UNESCO aumenta el número de visitantes. Este hecho entra en contradicción tanto con la integridad física del bien como con la propia filosofía de la UNESCO que en su documento de 1972 señala que los gestores deben salvaguardar la integridad del bien para las presentes y futuras generaciones (UNESCO, 1972). Por ello, se ha evidenciado un creciente interés en analizar qué impacto tiene la inclusión en la lista de WHS sobre diferentes variables: (1) la afluencia de visitantes (Landorf, 2009); (2) los ingresos y (3) la posible reducción de los controles y restricciones por temor a perder esos ingresos (Drost, 1996).

El aumento en el número de visitantes puede provocar aglomeraciones, sobre todo en época de temporada alta y tiene un impacto negativo tanto en los recursos patrimoniales como en la experiencia turística (Vaske et al., 1993; Vaske y Shelby, 2008). Esta circunstancia aumenta la relevancia de acometer una gestión con una visión intergeneracional y de sostenibilidad, que lleve al desarrollo e implementación de políticas para su uso sostenible e introduzca sistemas de gestión para conseguir mantener su integridad y autenticidad, todo ello, con el fin de que perduren para las generaciones futuras (Garrod y Fyall, 2000; Donato, 2011; Badia et al., 2012).

En este sentido, existe una necesidad de conversar, debatir y colaborar entre todas las partes implicadas para minimizar las amenazas al patrimonio (Bramwell y Lane, 1993; Mitchell y Reid, 2001; Aas et al., 2005; Graci, 2012). Si se puede encontrar una base común entre los grupos de interés, los recursos de las comunidades locales pueden ser preservados y el patrimonio turístico local sostenido (Parker, 1999; Tan et al., 2018).

Todo lo expuesto hasta ahora, pone de manifiesto la necesidad de gestionar el patrimonio cultural desde una perspectiva más empresarial, interdisciplinar y estratégica. Para que esto sea posible la gestión de un destino turístico concreto necesita de un planteamiento global focalizado en la satisfacción de turista (Laws, 1998) y en los principios de desa- 
rrollo sostenible, teniendo en cuenta los distintos agentes implicados en el proceso, sus objetivos que a veces entran en conflicto, y las propias particularidades organizativas de estos "edificios" (Bozány, 2007; UNESCO, 2014).

La UNESCO a partir de 2005 incorporó como requisitos para que un HS pudiese ser incluido en la lista de los WHS la implantación de un sistema de gestión vertebrado sobre un ciclo de planificación-control, que además permita la participación activa de los grupos de interés. Con esta aproximación holística e integrada se pretende conseguir un equilibrio entre la conservación, sostenibilidad y desarrollo del WHS.

Esta forma de afrontar la gestión de un WHS que conjuga el aspecto cultural con el económico, debe tener en la participación de los ciudadanos un punto de referencia básico, pues es condición básica para conseguir la generación de un valor económico para el territorio y la protección de los valores del patrimonio (Badia et al., 2012; UNESCO, 2014).

En el sistema de gobernanza o gestión (governance) juega un papel relevante el sujeto u organismo encargado de gobernar la red. Dicho sistema se debe construir sobre tres niveles (Badia et al., 2012):

a) Político-decisional. Supone la creación de un organismo cuyos componentes hayan sido elegidos por los ciudadanos y cuya principal misión es la de dirección estratégica y de control de los resultados sociales, culturales y económicos conseguidos por el WHS.

b) Técnico-organizativo. Relacionado con la creación de un sujeto que desempeñe un verdadero rol operativo en los procesos de desarrollo y sea el referente para todos los sujetos implicados.

c) Participativo, se refiere a la constitución de un organismo a través del cual se articule la participación de los ciudadanos y ejerza un control social. Este aspecto es muy importante para la UNESCO, pues los WHS deben jugar un importante valor identitario para la población local.

Landorf (2009) pone de manifiesto la escasez de estudios y trabajos en los que se aborde la consideración de la sostenibilidad en los planes de gestión de los WHS. A pesar de que esta es una cuestión de importancia universal, no sólo local o regional.

\section{5. "MANAGEMENT PLAN" DE LA UNESCO}

La necesidad de implementar sistemas reales de seguimiento en los WHS lleva a la UNESCO a considerar la gestión de los WHS como un campo de interés específico a principios de los 90 del pasado siglo. Ello supuso la elaboración de Management Guidelines for World Cultural Heritage Sites, en estas directrices se hace referencia a la necesidad de elaborar una estrategia integral de desarrollo turístico para cada uno de los sitios individuales patrimonio mundial. Sin embargo, dado que el objetivo principal de la Convención del Patrimonio Mundial es garantizar "la identificación, protección, conservación, presentación y transmisión a las generaciones futuras del patrimonio cultural y natural" (UNESCO, 1972), el énfasis de las directrices de gestión en sus inicios se ha 
centrado más en la conservación del patrimonio tangible que en la gestión del patrimonio inmaterial y la actividad de los visitantes (Rodwell, 2012).

La Operational Guidelines de 2005 señala que cada bien nominado a entrar en la lista UNESCO debe tener un "Management Plan" o documento similar el cual debe especificar cómo se van a tomar las acciones necesarias para salvaguardar el valor del "site" (Badia, 2011). Dicho plan debe permitir coordinar la legislación protectora, los mecanismos de gestión y las prácticas administrativas, todo ello, enlazado con el desarrollo sostenible de cada WHS, para preservar los sites para las generaciones futuras -equidad intergeneracional-.

En la última versión de la Operational Guidelines se continúa insistiendo en la necesidad de implantar un sistema de gestión efectiva diseñado bajo un ciclo de planificacióncontrol constante que incentive la participación de los stakeholder y contenga (UNESCOa; UNESCO, 2014):

- Una clara comprensión de quienes son todos los "propietarios". Haciendo mención expresa del uso de la planificación participativa y del proceso de consulta a los grupos de interés.

- Un ciclo de planificación, implementación, seguimiento evaluación y feedback.

- Evaluación de impacto de las vulnerabilidades de la propiedad a las presiones y cambios sociales, económicos y de otra índole, así como el seguimiento de los impactos de las tendencias e intervenciones propuestas.

- Desarrollo de mecanismos que fomenten la implicación y coordinación de todos los grupos de interés en la gestión.

- Asignación de los recursos necesarios para el desarrollo de la actividad.

- Gestión de la capacidad de asistencia del edificio.

- Sistema que informe de manera transparente de la gestión.

La expectativa de una aproximación completa e incorporable para conseguir “...un equilibrio apropiado y equitativo entre conservación, sostenibilidad y desarrollo", y asegurando “... la involucración activa de [...] las comunidades locales” aún está más enfatizado en la Declaración de Budapest del Patrimonio Mundial (UNESCO, 2002). Lamentablemente, apenas se facilita una guía para conseguir el objetivo citado (Wilson y Boyle, 2006; Langford,2009; Tan et al., 2018).

En cuanto a la estructura del plan de gestión, Landorf (2009), en seis estudios de caso realizados, comprobó que generalmente los WHS siguieron la estructura de tres partes propuesta por Feilden y Jokilehto (1998): descripción y delimitación del sitio, evaluación y objetivos, y administración general del sitio. En todos los planes analizados se proporciona una descripción detallada del sitio, los motivos de su inclusión en la Lista del Patrimonio Mundial y los marcos legales establecidos para garantizar su protección. Además, en todos ellos se identificaban diferentes cuestiones:

(1) Administración del sitio, planificación y política para establecer marcos de gestión a largo plazo. 
(2) Conservación como la base principal de las decisiones de gestión para el mantenimiento del valor universal excepcional de cada sitio.

(3) Desarrollo económico centrado en la regeneración económica del patrimonio.

(4) Acceso y gestión de visitantes incidiendo en la necesidad de estrategias de transporte sostenible, para gestionar el impacto del incremento de visitantes mediante la promoción del uso de bicicletas y del acceso peatonal al sitio.

(5) Conciencia pública, educación e investigación sobre los valores universales asociados con el WHS.

Badia (2011) señala que no se puede hablar de un único modelo para todos los WHS, que es mejor desarrollar guías de aplicación generales adaptables a cada caso concreto. Entiende que es necesario realizar un intercambio real entre todos los grupos de interés del lugar, en relación a la visión, misión y estrategias a implementar y que es necesario introducir sistemas de medida del rendimiento que consideren las dimensiones cualitativas y cuantitativas, que se enfoquen tanto en el corto como en el largo plazo y que sean multidimensionales, para ayudar a la gestión y rendición de cuentas a la comunidad.

En consecuencia, el éxito futuro de los sistemas de gestión del patrimonio depende en gran medida de que sean capaces de emplear un proceso basado en los valores, adoptar enfoques que prevean y gestionen el cambio e invertir en la relación entre el patrimonio y la sociedad, sopesando constantemente por qué y cómo debe conservarse el patrimonio cultural, para quién y con quién (UNESCO, 2014).

\section{MODELO PARA LA GESTIÓN SOSTENIBLE DE LOS WHS}

Con la intención de diseñar un modelo integral para la gestión sostenible de los WHS se ha adoptado una perspectiva amplia. Ésta contempla toda la información relevante para la gestión, entendiéndola como un proceso continuo de planificación y control para alcanzar los objetivos organizativos. Todo ello, considerando las características del entorno del WHS. Siguiendo a (Escobar y Lobo, 2002) se pueden distinguir tres partes:

A) Planificación o diseño del sistema.

B) Sistema Integral de Gestión Sostenible (SIGS) para los WHS.

C) Los Sistemas de Medición y Evaluación del rendimiento organizativo y de Retroalimentación.

En el modelo propuesto (ver figura 2) se adopta una aproximación holística, integradora y contingente que contempla todas las dimensiones. En concreto, las diferentes dimensiones de la sostenibilidad (medioambiental, económico-financiera, patrimonial y sociocultural) han sido analizadas de forma separada (Berthold et al., 2015).

Por otro lado, es muy importante prestar especial atención a la conceptualización del rendimiento organizativo en los WHS dada la naturaleza pública y no lucrativa de estas entidades. La propuesta incluye un conjunto de indicadores del rendimiento organizativo que no se limita a las variables cuantitativo-financieras, sino que incorpora otras de 
carácter cuantitativo no financiero y cualitativo que permiten medir y valorar el logro de los objetivos de los WHS en términos de sostenibilidad en un amplio sentido. El uso de indicadores de sostenibilidad constituye un buen punto de partida para el desarrollo del modelo (Berthold et al., 2015).

Además, de acuerdo con Longo (2005) para coordinar y dar participación activa a todos los grupos de interés involucrados el modelo se decanta por una red pivotante y la creación de una agencia encargada de la dirección y el control sobre el resto integrantes de la red, pero sin obedecer a un claro principio jerárquico top-down.

Finalmente, desde el punto de vista teórico, el sistema de medición-evaluación y retroalimentación, también conocido como sistema de control de gestión, se enmarca en la categoría de Control Político identificado por Hofstede (1981), dadas las características de la actividad de gestión de los WHS y la ambigüedad de objetivos que difícilmente puede ser resuelta. Esta ambigüedad se produce por un claro conflicto de intereses y valores percibidos por los diferentes grupos de interés, por la ausencia de conocimiento sobre la relación medios-fines y por un entorno cambiante. Para reducirla Hofstede (1981) propone:

- Utilización de la jerarquía: los niveles altos de autoridad o burocracia establecen los objetivos que para los niveles inferiores carecen de ambigüedad -políticas públicas y red pivotante-.

- Uso de reglas y políticas fijas; esto supone la elección arbitraria, pero se crea una apariencia de casi no ambigüedad.

- Uso de la negociación.

- Acudir a expertos que son percibidos como creíbles por parte de la organización.

- Control por crisis, siendo la situación de crisis la que supone una reducción de alternativas disminuyendo así la ambigüedad.

En relación al modelo se consideran las siguientes puntualizaciones:

A) Planificación o diseño del SIGS. En la parte superior del modelo se recogen el conjunto de variables que influirían de forma determinante en el diseño del sistema:

(1) Management Plan de la UNESCO. El diseño de este plan es una de las condiciones que cada HS debe cumplir para poder ser admitido en la Lista de WHS, por ello, las recomendaciones de la UNESCO deben ser parte del modelo propuesto.

(2) Políticas públicas. Las AAPP, además de ser propietarias de gran parte de los HS, son responsables de la aprobación de leyes de patrimonio y protección, de la fijación de políticas culturales, establecimiento de los principios y guías para la conservación y uso del patrimonio histórico-cultural, desarrollo e implementación de estrategias de desarrollo del turismo, inversiones en transportes e infraestructuras públicas, dotaciones presupuestarias y concesión de subvenciones, entre otras.

(3) Plan estratégico y objetivos organizativos. Sobre la premisa estratégica de la sostenibilidad del patrimonio tangible e intangible del WHS, el modelo adopta una visión multidisciplinar y una aproximación step by step que contemple un sistema multidimensional de objetivos divididos en acciones concretas para implementar la estrategia. Esta diversidad de objetivos a conseguir, a veces son difusos, ambiguos y contradictorios. 
Figura 2

MODELO PARA LA GESTIÓN SOSTENIBLE DE LOS WHS

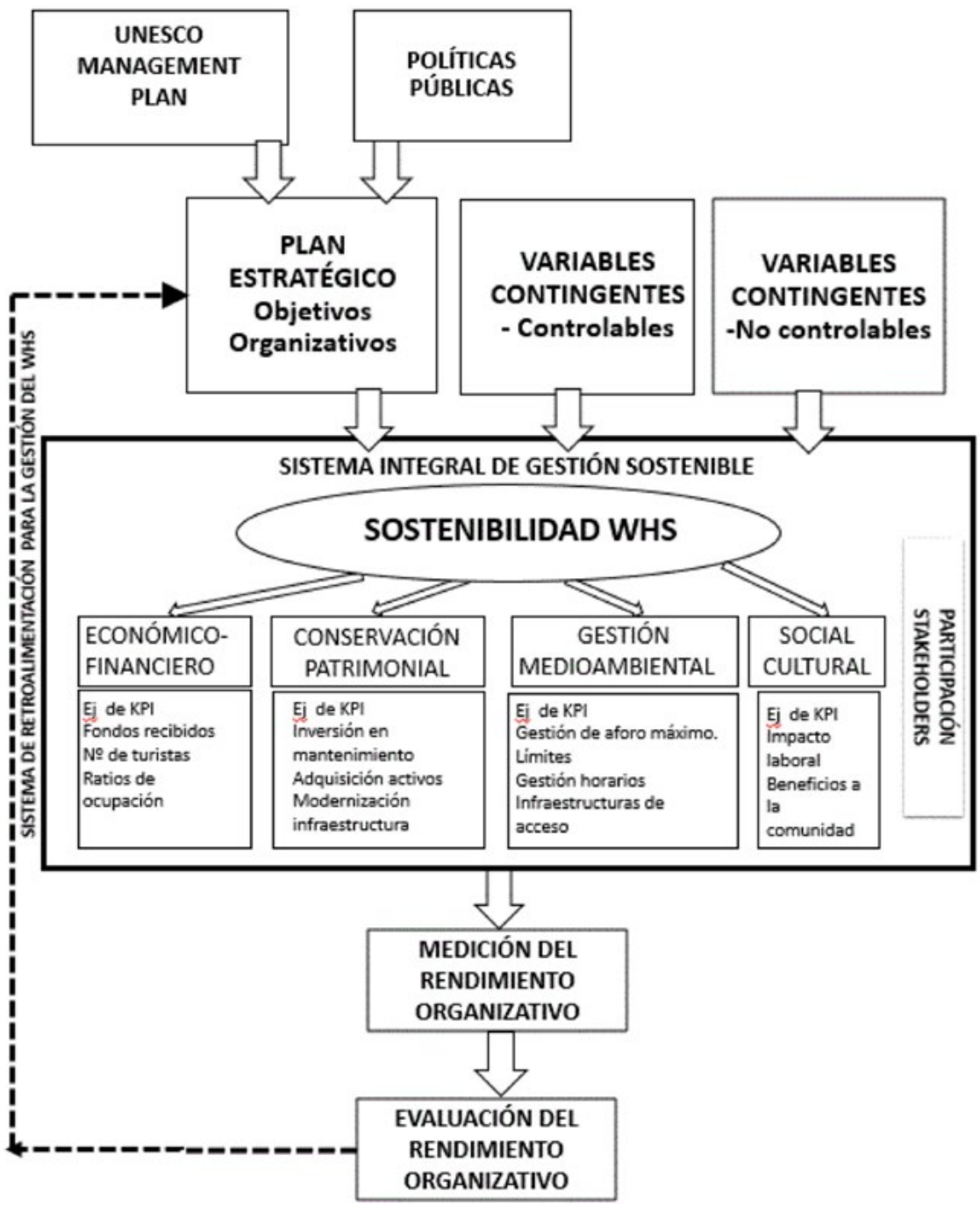

Fuente: Elaboración propia.

Según Badía (2011), los objetivos se deben agrupar como mínimo en las siguientes categorías: 
o Conservación patrimonial del sitio.

o Conservación urbanístico-paisajística.

o Económicos y de turismo.

o De impacto social.

(4) Variables contingentes controlables que serían aquellas sobre las que los gestores de los WHS podrían influir, por ejemplo, la limitación de visitas diarias, la fijación de precios del acceso, etc.

(5) Variables contingentes no controlables por los gestores de los WHS, son elementos externos como el nivel d e incertidumbre, inestabilidad y turbulencia del entorno político, legal y económico, tanto de la zona donde se sitúa el WHS como de otras ubicaciones, las características del sector en el que opera el WHS (grado de crecimiento, madurez, número de nuevos competidores, nivel y rapidez del desarrollo tecnológico del sector, etc.), la existencia de otros WHS en la zona.

B) Sistema de gestión integral sostenible (SGIS) para los WHS strictu sensu, en el que se incluyen las diferentes áreas de gestión relacionados con la sostenibilidad de los WHS:

(1) Gestión económico-financiera enfocada al desarrollo económico sostenible del WHS a largo plazo y de su zona.

(2) Gestión de la conservación patrimonial para el mantenimiento del valor universal excepcional de cada WHS, tangible e intangible.

(3) Gestión medioambiental como elemento fundamental del desarrollo turístico para el mantenimiento de los recursos naturales y de la diversidad biológica del entorno en el que opera el WHS.

(4) Gestión social para llevar a cabo actividades que tengan un impacto positivo a largo plazo en las comunidades locales de cada uno de los WHS. Ello conlleva la introducción de instrumentos que permitan la consecución de un consenso externo, que vayan más allá de la búsqueda de la eficiencia, eficacia y economicidad y que se fundamenten en la tutela y el respeto de los valores básicos de la comunidad, dando, cuando sea posible, voz y voto a los ciudadanos y a todos los grupos de interés.

Para hacer operativa la gestión según este SIGS propuesto sería también necesario el diseño de un conjunto de Key Performance Indicators (KPI) que permitirían cuantificar, de forma equilibrada para las cuatro grandes áreas de gestión, el rendimiento de los diferentes WHS y, en su caso, hacer posible la comparabilidad con otros WHS de la misma naturaleza con el objeto de desarrollar prácticas de benchmarking que permitiesen identificar las mejores prácticas de gestión sostenible e implantarlas para mejorar el rendimiento del WSH en el futuro.

Gilhespy (1999) identifica una serie de objetivos estratégicos tanto para los directivos como para los grupos de interés externos y para cada uno de ellos propone la elaboración de diversos indicadores de rendimiento: 


\section{Cuadro 1 \\ OBJETIVOS ESTRATÉGICOS E INDICADORES}

\begin{tabular}{|c|c|}
\hline \multicolumn{2}{|c|}{ Indicadores para los directivos } \\
\hline Objetivos estratégicos & Indicadores \\
\hline $\begin{array}{l}\text { Maximización del nivel de visita de } \\
\text { diferentes colectivos y equidad en el } \\
\text { acceso }\end{array}$ & $\begin{array}{l}\text { - Nuevos visitantes } / \mathrm{N}^{\circ} \text { total visitas } \\
\text { - Desempleados } / \mathrm{N}^{\circ} \text { total visitas } \\
\text { - Niños } / \mathrm{N}^{\circ} \text { total visitas } \\
\text { - Estudiantes } / \mathrm{N}^{\circ} \text { total visitas } \\
\text { - Visitantes locales } / \mathrm{N}^{\circ} \text { total visitas }\end{array}$ \\
\hline Nivel de asistencia & $\begin{array}{l}\text { - } \mathrm{N}^{o} \text { total visitas } \\
\text { - } \mathrm{N}^{o} \text { total visitas/Capacidad máxima } \\
\text { - } \mathrm{N}^{o} \text { total visitas/Visitas previstas }\end{array}$ \\
\hline Economía & $\begin{array}{l}\text { - Costes totales reales/Costes totales previstos } \\
\text { - Costes publicidad/Costes totales } \\
\text { - Costes totales/Ingresos totales }\end{array}$ \\
\hline Ingresos & $\begin{array}{l}\text { - Ingresos reales/Ingresos previstos } \\
\text { - Ingresos por subvenciones/Ingresos totales } \\
\text { - Ingresos por ventas entradas/Ingresos totales } \\
\text { - Ingresos totales } / \mathrm{N}^{\circ} \text { visitantes } \\
\text { - Ingresos totales } / \text { Gastos totales }\end{array}$ \\
\hline $\begin{array}{l}\text { Calidad del servicio y nivel de } \\
\text { excelencia }\end{array}$ & $\begin{array}{l}\text { - Realización de cuestionarios a visitantes } \\
\text { - Número de quejas y reclamaciones } \\
\text { - } \mathrm{N}^{\circ} \text { de accidentes de visitantes en el sitio } \\
\end{array}$ \\
\hline $\begin{array}{l}\text { Actividades educativas y culturales } \\
\text { (cursos, workshops, exposiciones) }\end{array}$ & $\begin{array}{l}\text { - } \mathrm{N}^{\mathrm{o}} \text { cursos, workshops, exposiciones... } \\
\text { - Costes educación/Costes totales } \\
\text { - Coste real de actuación/Coste previsto actuación }\end{array}$ \\
\hline \multicolumn{2}{|c|}{ Indicadores para los grupos de interés externos } \\
\hline Objetivos estratégicos & - Indicadores \\
\hline $\begin{array}{l}\text { Impacto económico y calidad de vida } \\
\text { en el territorio }\end{array}$ & $\begin{array}{l}\text { - Ingresos directos generados por el site } \\
\text { - Ingresos indirectos generados por el site } \\
\text { - } \mathrm{N}^{\mathrm{o}} \text { de empleos directos } \\
\text { - } \mathrm{N}^{\mathrm{o}} \text { de empleos indirectos } \\
\text { - Ocupación hotelera }\end{array}$ \\
\hline Prestigio local, nacional e internacional & $\begin{array}{l}\text { - Realización de encuestas } \\
\text { - Aumento del número de visitas }\end{array}$ \\
\hline
\end{tabular}

Fuente: Gilhespy (1999); (Towse, 2001); (Turbide y Laurin, 2009).

De todos ellos, como señala Paulus (2007), son más fáciles de definir y utilizar los indicadores cuantitativos que los cualitativos. Aunque esta afirmación la realiza para los museos, creemos que es trasladable a los WHS. 
En este sentido, en la Figura 2 hemos incluido algunos ejemplos de KPI para cada una de las cuatro grandes áreas de gestión identificadas en el modelo.

Por último, dada la relevancia del rol de todos los grupos de interés, éstos se han incluido en el modelo con la denominación de Participación de los Grupos de interés. Los gestores de los WHS han de llevar a cabo un proceso permanente y participativo de consulta a los grupos de interés mediante el empleo de mecanismos de interrelación vertical entre la agencia y las instituciones culturales individuales, y horizontal entre las instituciones individuales (proyectos conjuntos, centrales de compras, etc.).

C) Medición y evaluación del rendimiento organizativo y sistema de retroalimentación. Uno de los componentes más importantes del SIGS de los WHS sería el rendimiento organizativo, entendido como una variable clave dentro del modelo, ya que permitiría evaluar si es adecuado el grado de consistencia entre las diferentes variables de diseño del sistema (management plan, políticas públicas, objetivos organizativos y variables contingentes), por un lado, y del propio sistema de los WHS, por otro. El rendimiento y los objetivos organizativos son dos componentes del modelo propuesto íntimamente relacionados que tienen que estar presentes en los modelos de naturaleza contingente (Fisher, 1995; Fisher, 1998) para saber qué queremos y poder así compararlo con lo que está ocurriendo realmente.

El cuanto a la medición y de evaluación del rendimiento organizativo, decir que será el encargado de determinar la eficiencia y eficacia en el logro de los diferentes objetivos organizativos fijados para las cuatro grandes áreas de la gestión sostenible de los WHS. Este proceso se realizaría mediante la comparación entre los valores reales alcanzados y los fijados como objetivos para cada uno de los KPI en un determinado periodo de tiempo.

El Sistema de retroalimentación se encargará de distribuir la información en sentido inverso para que se emprendan las acciones recomendables para evitar que se produzcan las desviaciones detectadas en cada uno de los ciclos de gestión sostenible de los WHS. Este proceso pretende, en última instancia, la consecución de una mejora continua del rendimiento organizativo. Este sistema no sólo debe diseñarse y emplearse para contribuir a la concordancia entre los objetivos organizativos y el logro del rendimiento organizativo, sino que también tendría que contribuir al ejercicio de un control adaptativo que facilitase los inevitables procesos de cambio que han de afrontar los WHS en el futuro (Birnberg, 1998).

\section{CONSIDERACIONES FINALES Y FUTURAS EXTENSIONES}

La consideración del patrimonio cultural desde un punto de vista económico pone de manifiesto la necesidad de conjugar actuaciones encaminadas a su protección y conservación con otras que desarrollen y potencien su papel económico, por ello, entendemos que su gestión se debe afrontar desde una perspectiva de gestión empresarial sostenible.

Por otro lado, el desarrollo sostenible del turismo exige la participación informada de todos los agentes relevantes o grupos de interés, así como un liderazgo político firme para lograr una colaboración amplia y establecer un consenso (Landorf, 2009).

En este trabajo se ha realizado una propuesta de un modelo holístico, contingente e integrador para la gestión sostenible -planificación y control- de los WHS. El modelo 
no pretende ser universal, sino más bien un punto de referencia para su adaptación a las particularidades propias de cada WHS, puesto que como señala Badía (2011) no se puede diseñar un único y universal modelo para la totalidad de los WHS, que es mejor desarrollar guías de aplicación generales adaptables a cada caso concreto. Este modelo comprende los nueve elementos que según la UNESCO debe incluir cualquier sistema de gestión de un WHS (UNESCO, 2014).

Como resultado de la influencia de movimientos como la NGP y NGobP, se entiende que es básica la identificación clara de todos los grupos de interés implicados y el establecimiento de un mecanismo de participación de cada uno de ellos en relación a la visión, misión y estrategias a implementar. El modelo no puede obviar la introducción de sistemas de medida del rendimiento que considere las diferentes dimensiones de la gestión sostenible, en sus aspectos cualitativos y cuantitativos, que se enfoque tanto en el corto como en el largo plazo y que sea multidimensional, para ayudar a la gestión y rendición de cuentas a la comunidad. La consideración de todos los aspectos anteriormente mencionados hace necesario el uso estratégico de los sistemas de información en este tipo de organizaciones turísticas (Buhalis, 1998).

Todo ello, además supone que los sistemas de gestión que se introduzcan deben responder a las necesidades que plantea la consecución de la multiplicidad de objetivos a cumplir, por ello, deben ir más allá de los de naturaleza pública, centrados fundamentalmente en el control del gasto, pero al mismo tiempo tampoco pueden ser importados del sector privado directamente, ya que no se ajustan suficientemente a las características de estos nuevos modelos híbridos de gestión, que consideren de manera integral los diferentes factores mencionados anteriormente.

Como futuras extensiones de este trabajo se propone, en primer lugar, llevar a cabo diferentes estudios de caso en varios WHS, tanto españoles como de otros países, en los que se analice y contraste la relevancia que otorgan los gestores de WHS a los factores identificados en este trabajo, se determine el grado de desarrollo e implantación de los sistemas de control para la gestión en cada uno de los casos y se evalúe en qué medida los sistemas implantados responden al modelo propuesto. En segundo lugar, con los resultados obtenidos de los estudios de caso se propondría una versión mejorada del modelo teórico propuesto.

\section{BIBLIOGRAFÍA}

AAS, C., LADKIN, A. y FLETCHER, J. (2005): Stakeholder collaboration and heritage management", Annals of Tourism Research, vol. I (32), pp. 28-48.

ALAZAIZEH, M. M., HALLO, J. C., BACKMAN, S. J., NORMAN, W. C. y VOGEL, M. A. (2018): Giving voice to heritage tourists: indicators of quality for a sustainable heritage experience at Petra, Jordan, Journal of Tourism and Cultural Change. Disponible en: https://doi.org/10.1080/14766825.2018.1455693

ALAZAIZEH, M. M., HALLO, J. C., BACKMAN, S. J., WILLIAM, C. y VOGEL, M. A. (2016): Crowding standards at Petra Archaeological Park : a comparative study of McKercher's five types of heritage tourists Crowding standards at Petra Archaeological Park : a comparative, Journal of Heritage Tourism, vol. IV, nº11, pp. 364-381. 
ALEXANDROS, A. y JAFFRY, S. (2005): Stated preferences for two Cretan heritage attractions, Annals of Tourism Research, vol. IV, n³2, pp. 985-1005.

ASHWORTH, G. y PAGE, S. J. (2011): Urban tourism research: Recent progress and current paradoxes, Tourism Management, $\mathrm{n}^{\circ}$ XXXII, vol.1, pp. 1-15.

ASUAGA, C. y RAUSELL KÖSTER, P. (2006): Un Análisis de la gestión de instituciones culturales: el caso específico de los Museos, Revista Iberoamericana de Contabilidad de Gestión, vol. IV, no8, pp. 83-104.

BADIA, F. (2011): Contents and Aims of Management Plans for World Heritage Sites: Managerial Analysis with a Special Focus on the Italian Scenario. Encatc Journal of Cultural Management and Policy. Disponible en: https://www.encatc.org/media/2708journal_vol1_issue1_dec20114150.pdf

BADIA, F. y DONATO, F. (2013): Performance measurement at world heritage sites: Per Aspera Ad Astra, International Journal of Arts Management, vol. I, nº16, pp. 20-34.

BADIA, F., DONATO, F. y GILLI, E. (2012): Profili economici e manageriali per la governance delle istituzioni culturali: il caso dei siti UNESCO, Annali Dell'Università Degli Studi Di Ferrara. Disponible en: http://annali.unife.it/museologia/article/viewFile/388/336

BARALDI, S. B. (2014): Evaluating results of public sector reforms in rechtsstaat countries: The role of context and processes in the reform of the Italian and French cultural heritage system, International Public Management Journal, vol. III, nº17, pp. 411-432.

BERTHOLD, É., RAJAONSON, J. y TANGUAY, G. A. (2015): Using sustainability indicators for Urban Heritage management: a review of 25 case studies, International Journal of Heritage and Sustainable Development, vol. XXII, nº4, pp. 23-34

BIRNBERG, J. G. (1998): Some Reflections on the Evolution of Organizational Control, Behavioral Research in Accounting, $\mathrm{n}^{\circ} \mathrm{X}$, Supl., pp. 27-40

BONET, L. y DONATO, F. (2011): The Financial Crisis and its Impact on the Current Models of Governance and Management of the Cultural Sector in Europe, Encatc Journal of Cultural Management and Policy. Disponible en: https://www.encatc.org/ media/2703-journal_vol1_issue1_dec2011512.pdf

BOZÁNY, A. (2007): Heritage site area type classification for facility management purposes, Journal of Facilities Management, vol. I, n5, pp. 62-77.

BRAMWELL, B. y LANE, B. (1993): Sustainable tourism: An evolving global approach, Journal of Sustainable Tourism, vol. I, $\mathrm{n}^{\circ} 1$, pp. 1-5.

BRAMWELL, B. y LANE, B. (2011): Critical research on the governance of tourism and sustainabilityJournal of Sustainable Tourism, vol. IV-V, nº19, pp. 411-421.

BRITO RODRÍGUEZ, M. y CÀNOVES VALIENTE, G. (2019): El desarrollo turístico en Mazatlán, México: un análisis de las condiciones de la sostenibilidad, Cuadernos de Turismo, no43, pp. 187-213, DOI: http://dx.doi.org/10.6018/turismo.43.08

BRUNDTLAND, G. (1987): Report of the World Commision on Environement and Development: Our Common Future, Oxford Paperbacks, Report of, 400. https://doi. org/10.2307/2621529. Disponible en http://www.un-documents.net/wced-ocf.htm.

BUHALIS, D. (1998): Strategic Use of Information Technologies in the Tourism IndustryTourism Management, vol. V, nº19, pp. 409-421. 
CASTRO LEÓN, J. F. (2005): La Calidad como herramienta de gestión del Turismo Cultural, Pasos. Revista de Turismo y Patrimonio Cultural, n III, vol.1, pp. 143-148. COLBERT, F. (2014): The Arts Sector: A Marketing Definition, Psychology and Marketing, vol. VIII, $\mathrm{n}^{\circ} 3$, pp. 563-565.

DIMAGGIO, P. J. y POWELL, W. W. (1983): The Iron Cage Revisited: Institutional Isomorphism and Collective Rationality in Organizational FieldsAmerican Sociological Review, vol. II, n48, pp. 147-160.

DONATO, F. (2011): Un approccio “multi-scala" per la gestione del patrimonio culturale italianoIl Capitale Culturale, $\mathrm{n}^{\circ} 2$, pp. 197-225.

DROST, A. (1996): Developing sustainable tourism for world heritage sitesAnnals of Tourism Research, vol. II, n²3, pp. 479-484.

DU CROS, H., BAUER, T., LO, C. y RUI, S. (2005): Cultural Heritage Assets in China as Sustainable Tourism Products: Case Studies of the Hutongs and the Huanghua Section of the Great Wall, Journal of Sustainable Tourism, vol. II, nº13, pp. 171-194.

ESCOBAR PÉREZ, B. y LOBO GALLARDO, A. (2002): El impacto de las adquisiciones transnacionales sobre los sistemas de control: el caso de TESARevista Española de Financiación y Contabilidad, vol. CXIII, n³1, pp. 725-765.

ETC/WTO (2005): City tourism and culture the European Experience, European Travel Commision. Disponible en https://doi.org/10.18111/9789284409150

FEILDEN, B.M. y JOKILEHTO, J. (1998): Management Guideliness for World Cultural Heritage Sites, ICCROM, UNESCO. Disponible en https://www.iccrom.org/publication/management-guidelines-world-cultural-heritage-sites.

FISHER, J. (1995): Contingency-based research on management control systems: Categorization by level of complexity, Journal of Accounting Literature, n ${ }^{\circ} 14$, pp. 24-53.

FISHER, J. G. (1998): Contingency Theory, Management Control Systems and Firm Outcomes: Past Results and Future DirectionsBehavioral Research in Accounting, $\mathrm{n}^{\circ} 70$ (supplement), pp. 47-64.

FRESNEDA FUENTES, S. y LOBO GALLARDO, A. (2014): Una aproximación a los factores claves para la gestión de los bienes de patrimonio cultural, Tourism and Management Studies, ${ }^{\circ} 10$ (special), pp. 186-192.

GARROD, B. y FYALL, A. (2000): Managing heritage tourismAnnals of Tourism Research, vol. III, n'27, pp. 682-708.

GILHESPY, I. (1999): Measuring the performance of cultural organizations: A model International Journal of Arts Management, vol.I, n², pp. 38-52.

GRACI, S. (2012): Collaboration and Partnership Development for Sustainable TourismTourism Geographies, vol. I, nº 15, pp. 25-42.

HERNÁNDEZ, F. (2002): El patrimonio cultural: la memoria recuperada. Gijón, TREA.

HO, P. S. Y. y MCKERCHER, B. (2004): Managing heritage resources as tourism productsAsia Pacific Journal of Tourism Research, vol. III, nº, pp. 255-266.

HOFSTEDE, G. (1981): Management control of public and not-for-profit activitiesAccounting, Organizations and Society, vol. III, n6, pp. 193-211.

HOOD, C. (1995): The "new public management" in the 1980s: Variations on a themeAccounting, Organizations and Society, vol. II-III, n²0, pp. 93-109. 
HUA, S. (2010): World Heritage Classification and Related Issues - A Case Study of the "Convention Concerning the Protection of the World Cultural and Natural Heritage, Procedia Social and Behavioral Sciences, $\mathrm{n}^{\circ} 2$, pp. 6954-6961.

INE (2017):Contabilidad Nacional Trimestral de EspañaBase 2010 Cuarto trimestre de 2016. Nota de prensa. Disponible en https://www.ine.es/prensa/cntr0117.pdf.

KAPLAN, R. y NORTON, D. (2001): Transforming the balanced scorecard from performance measurement to strategic management : Part IAccounting Horizons, vol. I, $\mathrm{n}^{\circ} 15, \mathrm{pp} .87-104$.

KAPLAN, R. y NORTON, D. (2001a): Transforming the balanced scorecard from performance measurement to strategic management : Part IIAccounting Horizons, vol. II, $\mathrm{n}^{\circ} 15, \mathrm{pp} .147-160$.

LAFORTUNE, A., ROUSSEAU, J.-G. y BÉGIN, L. (1999): An Exploration of Management Control in the Arts and Cultural SectorInternational Journal of Arts Management, vol. I, no2, pp. 64-76.

LANDORF, C. (2009): Managing for sustainable tourism: a review of six cultural World Heritage SitesJournal of Sustainable Tourism, vol. I, nº17, pp. 53-70.

LARA DE VICENTE, F. y LÓPEZ-GUZMÁN GUZMÁN, T. J. (2004): El turismo como motor de desarrollo económico en ciudades patrimonio de la humanidad Pasos. Revista de Turismo y Patrimonio Cultural, n², pp. 243-256.

LAWS, E. (1998): Conceptualizing visitor satisfaction management in heritage settings: an exploratory blueprinting analysis of Leeds Castle, KentTourism Management, vol. VI, n' 19 , pp. 545-554.

LONGO, F. (2005): Governance dei network di pubblico interese.Milano, Egea.

MILLAR, S. (1989): Heritage management for heritage tourism Tourism Management, vol. I, no10, pp. 9-14.

MITCHELL, R. E. y REID, D. G. (2001): Community integration: Island tourism in PeruAnnals of Tourism Research, vol. XI, n²8, pp. 113-139.

PADDISON, B. y WALMSLEY, A. (2018): New Public Management in tourism: a case study of YorkJournal of Sustainable Tourism, vol. VI, n 26, pp. 1-17.

PARKER, S. (1999): Developing a typology of sustainable tourism partnershipsJournal of Sustainable Tourism, vol. III-IV, $\mathrm{n}^{\circ} 7$, pp. 260-273

PASTOR ALFONSO, M. J. (2003): El patrimonio cultural como opción turística, Horizontes Antropológicos, vol. XX, nº9, pp. 97-115.

PAULUS, O. (2007): Measuring museum performance: A Study of museums in France and the United StatesRecent Developments in Cultural Economics, vol.I, nº, pp. 50-63.

PONS, A., RODERS, A. R. P. y TURNER, M. (2011): The sustainability of management practices in the Old City of Salamanca, Facilities, vol. VII-VIII, n²9, pp. 326-338.

RODWELL, D. (2012): The Unesco World Heritage Convention, 1972-2012: Reflections and DirectionsThe Historic Environment: Policy \& Practice, vol. I, n³, pp. 64-85.

RUNYA, X., QIGUI, S. y WEI, S. (2015): The Third Wave of Public Administration: The New Public Governance, Canadian Social Science, vol. VII, nº11, pp. 11-21.

SCHUSTER, J. M. (1996): The performance of performance indicators in the arts, Nonprofit Management and Leadership, vol. III, nº7, pp. 253-269. 
SU, R., BRAMWELL, B. y WHALLEY, P. A. (2018): Cultural political economy and urban heritage tourismAnnals of Tourism Research, ${ }^{\circ} 68$, pp. 30-40.

TAN, S. K., TAN, S. H., KOK, Y. S. y CHOON, S. W. (2018): Sense of place and sustainability of intangible cultural heritage - The case of George Town and MelakaTourism Management, $\mathrm{n}^{\circ} 67$, pp. 376-387.

TAJTÁKOVÁ, M. (2006): Flexibility of strategic choices in opera house managementInternational Journal of Business Environment, n⿳3, pp. 365-381.

TOWSE, R. (2001): The Case of the Royal Opera House , Covent Garden Quis custodiet ? the Or Managing Management International Journal of Arts Management, vol. III, no3, pp. 38-50.

TURBIDE, J. y LAURIN, C. (2009): Performance Measurement in the Arts Sector: The Case of the Performing ArtsInternational Journal of Arts Management, vol. II, $\mathrm{n}^{\circ} 11$, pp. 56-70.

ULLDEMOLINS, J. R. y AROSTEGUI, A. R. (2013): The governance of national cultural organisations: Comparative study of performance contracts with the main cultural organisations in England, France and Catalonia (Spain)International Journal of Cultural Policy, vol. II, nº19, pp. 1-21.

UNESCO (1972): Convention Concerning the Protection of the World Cultural and Natural HeritagGeneral Conference Seventeenth Session, 1(November), pp. 135-145.

UNESCO: Disponible en http://www.unesco.org/education/tlsf/mods/theme_c/mod16. html.

UNESCOa: The Operational Guidelines for the Implementation of the World Heritage Convention. Disponible en https://whc.unesco.org/en/guidelines/.

UNESCO (2002): 26th session of the World Heritage Committee, Budapest, Hungary, 24-29 June 2002. Disponible en http://whc.unesco.org/en/sessions/26com/documents/.

UNESCO (2014): Gestión del patrimonio mundial cultural. Manual de referencia. Paris, UNESCO.

UNESCO (2017): Operational Guidelines for the Implementation of the World Heritage Convention, Intergovernmental Committee for the Protection of the World Cultural and Natural Heritage. Disponible en: http://www.bcin.ca/Interface/openbcin.cgi?submit=s ubmit\&amp;Chinkey=148022.

UNWTO: Sustainable Development of Tourism. Disponible en http://sdt.unwto.org/es/ content/definicion.

VASKE, J. J., DONNELLY, M. P. y SHELBY, B. (1993): Establishing management standards: Selected examples of the normative approachEnvironmental Management, vol. $\mathrm{V}, \mathrm{n}^{\mathrm{o}} 17, \mathrm{pp} .629-643$.

VASKE, J. J. y SHELBY, L. B. (2008): Crowding as a descriptive indicator and an evaluative standard: Results from 30 years of researchLeisure Sciences, n³0, pp. 111-126.

WELFORD, R., YTTERHUS, B. y ELIGH, J. (1999): Tourism and sustainable development: An analysis of policy and guidelines for managing provision and consumptionSustainable Development, vol IV, nº, pp. 165-177.

WILSON, L. y BOYLE, E. (2006): Interorganisational collaboration at UK World Heritage SitesLeadership \& Organization Development Journal, vol. VI, n²7, pp. 501-523. 
WTO (2019): All Countries: Tourism industries: indicators 1995-2017, Tourism Statistics (1). Disponible en https://www.e-unwto.org/doi/abs/10.5555/ unwtotfb0000301019952017201901.

ZAN, L. (2006): Managerial Rhetoric and Arts Organizations. New York, Palgrave Mcmillan. 